\title{
Macular Microvasculature Density Changes in Anisometropic Amblyopic Eyes after Successful Treatment
}

\author{
Wuhe Chen $\mathbb{D}^{1},{ }^{1}$ Frank Thorn, ${ }^{1,2}$ Ruzhi Deng, ${ }^{1}$ Xiaoman Li, ${ }^{1}$ Jiangtao Lou $\mathbb{D},{ }^{3}$ \\ Yingjie Wang $\left(\mathbb{C}^{3},{ }^{3}\right.$ and Xinping $\mathrm{Yu} \mathbb{1}^{1}$ \\ ${ }^{1}$ School of Ophthalmology and Optometry, Eye Hospital of Wenzhou Medical University, Wenzhou 325000, Zhejiang, China \\ ${ }^{2}$ Department of Brain and Cognitive Sciences, Massachusetts Institute of Technology, Cambridge 02139, MA, USA \\ ${ }^{3}$ School of Ophthalmology and Optometry, Wenzhou Medical University, Wenzhou 325000, Zhejiang, China
}

Correspondence should be addressed to Xinping Yu; yu-xinping@163.com

Received 23 August 2020; Revised 13 October 2020; Accepted 22 October 2020; Published 9 November 2020

Academic Editor: David P. Piñero

Copyright (c) 2020 Wuhe Chen et al. This is an open access article distributed under the Creative Commons Attribution License, which permits unrestricted use, distribution, and reproduction in any medium, provided the original work is properly cited.

Purpose. To determine if the abnormal macular microvasculature in hyperopic anisometropic amblyopia changes after occlusion therapy, and to discover if the macular microvasculature influences the efficacy of amblyopic treatment. Materials and Methods. Twenty-two children with hyperopic anisometropic amblyopia (median, 8 years old) were enrolled along with 66 healthy controls of similar age and gender distribution. Best-corrected visual acuity (BCVA) was determined, and macular vessel density in the superficial and deep capillary plexuses (SCPs and DCPs, respectively) was measured by optical coherence tomography angiography before and after refractive correction and occlusion therapy. Changes in BCVA and macular SCP and DCP vessel density were measured after amblyopia treatment of $7.5 \pm 4.4$ months. Results. The amblyopic baseline BCVA, logMAR $0.42 \pm 0.27$, improved to $\log$ MAR $0.18 \pm 0.18$ in 14 patients $(64 \%)$ after amblyopia treatment $(P<0.001)$. In 8 patients $(36 \%)$, there was no improvement in the BCVA. In amblyopic eyes with improved BCVA, the SCP density tended to increase in each parafoveal quadrant, while it tended to decrease in all quadrants of the fellow eyes. For patients in whom BCVA did not improve, the macular SCP vessel density tended to decrease in all quadrants of the amblyopic and fellow eyes. The posttreatment difference in SCP vessel density between amblyopic and fellow eyes was not significant. Multiple linear regression analysis showed that older age and greater foveal SCP vessel density were negatively correlated with BCVA improvement $(P<0.018$ and $P<0.036$, respectively). Conclusions. Macular vessel density tends to increase in anisometropic amblyopic eyes after successful treatment. Younger age and lower SCP macular density are predictive of a greater therapeutic effect of occlusion therapy.

\section{Introduction}

Amblyopia commonly causes life-long reduced vision in children, affecting $1.6 \%$ to $3.6 \%$ of the population [1]. Both functional and morphological changes involved in amblyopic development are known to occur in the central visual pathway. Extracellular recordings from striate neurons of monkeys with amblyopia show that most striate neurons respond from the sound eye and only a few respond from the amblyopic eye [2]. In addition, there is shrinkage of cells in the lateral geniculate nucleus (LGN) of the amblyopic eye [3]. The involvement of the retina in amblyopia has always been inconclusive and controversial. However, with recent advances in imaging and other technologies, the involvement of the macula has been more thoroughly evaluated, and retinal changes associated with amblyopia have been reported.

Recently, several studies have used optical coherence tomography (OCT) to evaluate macular thickness in the amblyopic eye. Although the results are not consistent, most recent studies show that there are differences in macular structure between amblyopic and normal eyes. Several groups found that the macula was thicker in anisometropic and strabismic amblyopia [4-6], while Nishi et al. [7] reported that the length of the photoreceptor outer segments was significantly greater in the fellow eyes than in the 
amblyopic eyes. Especially relevant to the present study, Nishi et al. [7] evaluated macular retinal microstructure during optical treatment in children with hyperopic anisometropic amblyopia and discovered an increase in the photoreceptor outer segment lengths in the fovea after treatment. This suggests a more mature macular structure as vision improves.

With the development of OCT angiography (OCTA), several studies evaluated macular vessel density in the superficial and deep capillary plexuses (SCPs and DCPs, respectively) of amblyopic eyes. Demirayak et al. reported that there were no differences in the macular vessel density of the SCP and DCP between amblyopic eyes, controls, and fellow eyes of patients with unilateral amblyopia [8]. However, more studies reported reduced macular vessel density of amblyopic eyes [9-13]. Chen et al. [9], from our clinic, also showed lower vascular density in the macular SCP of strabismic and bilateral amblyopic eyes and markedly lower density in anisometropic amblyopic eyes. In addition, macular vessel density was positively correlated with retinal thickness. Based on these observations, we hypothesized that the macular microvasculature might also be altered as vision improves. To the best of our knowledge, there has been no longitudinal study to measure the effect of amblyopia treatment on macular microvasculature in children with amblyopia.

The purpose of this study was to determine if the abnormal macular SCP microvasculature density in hyperopic anisometropic amblyopia changes in conjunction with improved BCVA during occlusion therapy. Also, we sought to determine if the macular microvasculature density might influence the efficacy of amblyopia treatment because this information could assist clinicians in the prediction of treatment outcomes.

\section{Materials and Methods}

2.1. Subjects. This prospective, longitudinal study was conducted between February 1, 2018, and February 28, 2019, at the Eye Hospital of Wenzhou Medical University, Wenzhou, Zhejiang, China. The protocol was approved by the Ethics Board of the Wenzhou Medical University and followed the tenets of the Declaration of Helsinki for research involving human subjects. Informed consent forms were explained to parents, who then signed and returned them to the researchers before examinations were performed on their child.

Hyperopic anisometropic amblyopia $(N=22)$ and agematched normal control children $(N=66)$ participated in the study. Amblyopia was defined by the American Academy of Ophthalmology's Preferred Practice Pattern (PPP) [14] as the best-corrected visual acuity (BCVA) $\geq 2$ lines of interocular difference. Inclusion criteria were unilateral amblyopia with a hyperopic refractive difference greater than 2.00 diopters (D), age between 6 and 12 years, and central fixation as determined by ophthalmoscopy. The control children's visual acuity was equal to or better than 20/20 in both eyes. The exclusion criteria included myopia greater than $-0.50 \mathrm{D}$, neurological diseases, and ocular conditions such as glaucoma or retinal disorders, nystagmus, strabismus surgery, and evidence-based visual therapy.

All subjects had an initial comprehensive ophthalmologic examination, including visual acuity with standard logarithmic visual acuity tumbling E charts, subjective refraction (after cycloplegia for amblyopic children), fundoscopy, slit-lamp examination, and an orthoptic evaluation. Axial length (AL) was measured with the Lenstar S 900 biometer (Haag-Streit AG, Koeniz, Switzerland). Macula vascular density was measured by OCTA (Optovue, Inc, Freemont, CA, USA). All amblyopic children underwent treatment with refractive correction and part-time patching of the fellow eye according to the American Academy of Ophthalmology's PPP [14]: 2 hours/day for moderate amblyopia (20/40 to 20/80) and 6 hours/day for severe amblyopia (20/100 to 20/400). Amblyopic children were followed up every three months, and AL and vascular density were measured at every visit.

2.2. OCTA Data Acquisition and Processing. Macular scans were taken using a spectral-domain OCTA system with AngioVue software (Optovue, Inc.), as described in previous publications $[15,16]$. The light source was $840 \mathrm{~nm}$ with a bandwidth of $45 \mathrm{~nm}$, and the A-scan rate was 70,000 scans per second. The AngioVue retina $(3 \times 3 \mathrm{~mm})$ procedure (Optovue, Inc.) was performed for both eyes.

Macular OCTA scans $(3 \times 3 \mathrm{~mm})$ consisted of two repeated B-scans at 304 raster positions, with 304 A-scans for each B-scan. Two volumetric raster scans were taken consecutively, one with a horizontal and the other with a vertical priority. Each scan was automatically segmented to visualize the macular SCP and DCP. SCP images were limited within an inner boundary at the internal limiting membrane and an outer boundary at $10 \mu \mathrm{m}$ above the inner plexiform layer. DCP images were limited by an inner boundary $10 \mu \mathrm{m}$ above the inner plexiform layer and an outer boundary $10 \mu \mathrm{m}$ below the outer plexiform layer. The 3D projection artifacts removal algorithms (Optovue, Inc.) were implemented in the AngioVue software to remove "false" blood flow signals from the DCP.

Vessel densities were determined in areas within each retinal location of interest. The five measured regions were based on the Early Treatment Diabetic Retinopathy Study regions and included the fovea and the temporal, superior, nasal, and inferior quadrants of the parafovea. The foveal region was a $1 \mathrm{~mm}$ diameter circle centered on the fovea. The parafoveal region was an annulus with a $3 \mathrm{~mm}$ outer diameter and a $1 \mathrm{~mm}$ inner diameter centered at the fovea (Figure 1). Quantitative analyses of the vessel density in the SCP and DCP were performed using the AngioVue automated software. The AngioVue automated software also calculated the area $\left(\mathrm{mm}^{2}\right)$ of the foveal avascular zone (FAZ). We reviewed all scans for correct segmentation and adequate image quality. Poor-quality scans (quality index $<8)$ were excluded from analysis.

2.3. Statistical Analyses. Statistical analyses were performed with SPSS software (version 23.0; SPSS Inc., Chicago, IL, USA). Means and standard deviations were used to describe 


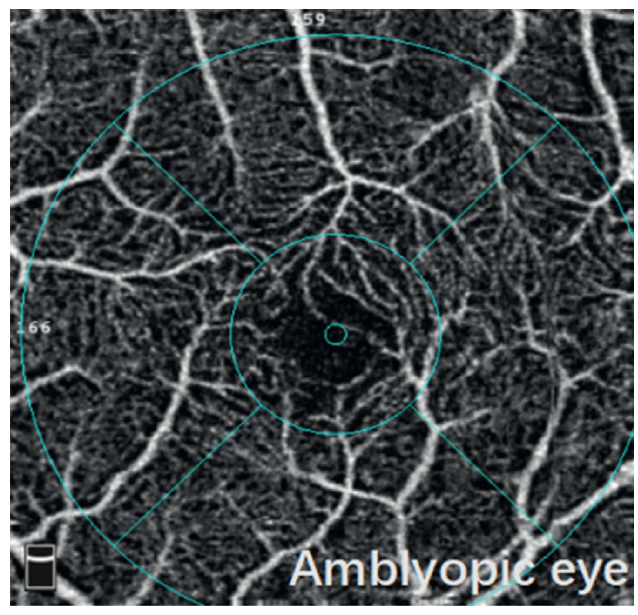

(a)

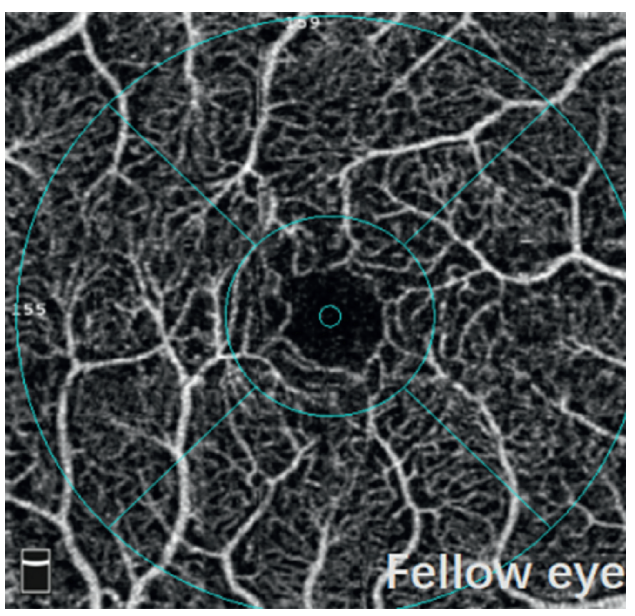

(b)

Figure 1: Optical coherence tomographic angiography scans of the macular microvasculature. Representative central $3 \times 3 \mathrm{~mm}$ macular scans of the superficial capillary plexus from an (a) amblyopic eye and (b) fellow eye. Macular vessel density was separately calculated for the fovea and the parafovea as well as the temporal, superior, nasal, and inferior parafoveal quadrants based on the Early Treatment Diabetic Retinopathy Study contour.

TABle 1: Demographics and biometrics of patients and controls at baseline.

\begin{tabular}{|c|c|c|c|c|c|c|}
\hline \multirow{2}{*}{ Variables } & \multicolumn{6}{|c|}{$P$ value } \\
\hline & Amblyopic eyes & Fellow eyes & Control eyes & Amblyopia vs. fellow & Amblyopia vs. control & Fellow vs. control \\
\hline Age (median, IQR), years & $8(7,9)$ & $8(7,9)$ & $8(7,9)$ & $>0.999$ & 0.943 & 0.943 \\
\hline Gender (boy/girl) & $9 / 13$ & $9 / 13$ & $39 / 27$ & $>0.999$ & 0.149 & 0.149 \\
\hline VA (LogMAR) & $0.42 \pm 0.31$ & $0.005 \pm 0.03$ & $0.00 \pm 0.03$ & $<0.001$ & $<0.001$ & 0.823 \\
\hline $\mathrm{SE}($ mean $\pm \mathrm{SD}), \mathrm{D}$ & $4.44 \pm 1.89$ & $0.70 \pm 0.87$ & $0.16 \pm 0.33$ & $<0.001$ & $<0.001$ & 0.008 \\
\hline $\mathrm{AL}($ mean $\pm \mathrm{SD}), \mathrm{mm}$ & $21.75 \pm 0.96$ & $23.05 \pm 0.90$ & $23.19 \pm 0.74$ & $<0.001$ & $<0.001$ & 0.507 \\
\hline
\end{tabular}

IQR: interquartile range; LogMAR: logarithm (minimum angle of resolution); SE: spherical equivalent; D: diopter; AL: axial length; SD: standard deviation.

continuous variable distributions. BCVA data were converted to logarithm (minimal angle of resolution) (logMAR) format for statistical analyses. The significance of vascular density differences between each subject's two eyes was compared by paired $t$-tests. Analysis of covariance (ANCOVA) adjusted for AL, age, and gender was used to evaluate vascular density differences between both eyes of amblyopic subjects and control eyes. A two-dimensional factorial repeated-measures ANOVA (before and after treatment of $x$ amblyopic eye and fellow eye) was used to determine if macular vessel density in amblyopic and fellow eyes differed before and after amblyopia treatment. Independent $t$-tests and Mann-Whitney tests were used to compare age, refraction, BCVA, duration of treatment, and macular vessel density in subjects with and without BCVA improvement. The association of age, refraction, BCVA, duration of treatment, and macular vessel density with the BCVA improvement was also examined by multiple linear regression analyses. Two-sided tests with $P<0.05$ were considered statistically significant.

\section{Results}

The median age of both the amblyopic subjects and the control subjects was 8 years with an interquartile range (IQR) of 7 to 9 years, and there was no difference between the amblyopic and control groups $(P=0.943$, Man$\mathrm{n}$-Whitney test). For control subjects, the data from the right eye were selected for analysis. As expected, spherical equivalent (SE), AL, and BCVA differed significantly between the amblyopic and fellow eyes and between the amblyopic and control eyes $(P<0.0001$ for all comparisons, Table 1$)$. There was no significant difference in BCVA $(P=0.823$, independent $t$-test $)$ or $\mathrm{AL}$ $(P=0.507)$ between the fellow and control eyes; however, the SE of the fellow eyes, $0.70 \pm 0.87 \mathrm{D}$, was slightly more hyperopic than the control eyes, $0.16 \pm 0.33 \mathrm{D}(P=0.008$, Table 1).

Vessel density of the macular SCP and DCP was measured by OCTA in amblyopic and control subjects before treatment (Table 2). Foveal SCP vessel density in the amblyopic eyes was less than in the fellow and control eyes, but the differences were not significant $(P>0.111)$. The parafoveal SCP vessel density in amblyopic eyes, $49.0 \pm 4.1 \%$, was less than in fellow eyes, $51.8 \pm 2.2 \%(P=0.012$, paired $t$-test $)$ and control eyes, $51.7 \pm 2.6 \%(P=0.010$, ANOVA $)$. The parafoveal SCP vessel densities in temporal, superior, and nasal quadrants were less in the amblyopia eyes than in fellow eyes $(P=0.003,0.036$, and 0.015 , respectively). Similarly, the parafoveal SCP vessel densities of the temporal and inferior quadrants of the amblyopic eyes were less than in the control eyes ( $P=0.002$ and 0.030 , respectively). There 
TABLE 2: Macular vessel density in amblyopic and the normal control group before treatment.

\begin{tabular}{|c|c|c|c|c|c|c|}
\hline \multirow{2}{*}{ Retinal region } & \multicolumn{3}{|c|}{ Macular vessel density, mean \pm SD (\%) } & \multicolumn{3}{|c|}{$P$ value } \\
\hline & Amblyopic eyes & Fellow eyes & Control eyes & Amblyopia vs. fellow & Amblyopia vs. control & Fellow vs. control \\
\hline \multicolumn{7}{|l|}{ SCP } \\
\hline Fovea & $23.8 \pm 4.8$ & $24.6 \pm 5.8$ & $25.3 \pm 5.5$ & 0.200 & 0.111 & 0.474 \\
\hline Parafovea & $49.0 \pm 4.1$ & $51.8 \pm 2.2$ & $51.7 \pm 2.6$ & 0.012 & 0.010 & 0.840 \\
\hline $\mathrm{T}$ & $47.6 \pm 4.6$ & $50.8 \pm 2.5$ & $50.8 \pm 2.8$ & 0.003 & 0.002 & 0.980 \\
\hline$S$ & $50.3 \pm 4.0$ & $52.6 \pm 2.9$ & $52.4 \pm 2.9$ & 0.036 & 0.155 & 0.905 \\
\hline $\mathrm{N}$ & $48.2 \pm 4.4$ & $51.2 \pm 2.1$ & $51.0 \pm 3.4$ & 0.015 & 0.057 & 0.914 \\
\hline $\mathrm{I}$ & $50.2 \pm 4.5$ & $52.4 \pm 2.7$ & $52.7 \pm 3.0$ & 0.104 & 0.030 & 0.481 \\
\hline \multicolumn{7}{|l|}{$\overline{\mathrm{DCP}}$} \\
\hline Fovea & $32.1 \pm 6.1$ & $32.5 \pm 6.5$ & $34.2 \pm 5.9$ & 0.684 & 0.141 & 0.703 \\
\hline Parafovea & $51.6 \pm 4.3$ & $52.9 \pm 2.7$ & $53.0 \pm 3.7$ & 0.221 & 0.289 & 0.265 \\
\hline $\mathrm{T}$ & $52.0 \pm 3.9$ & $52.6 \pm 3.5$ & $53.2 \pm 3.7$ & 0.594 & 0.138 & 0.602 \\
\hline S & $51.5 \pm 5.2$ & $53.1 \pm 3.4$ & $53.3 \pm 3.8$ & 0.213 & 0.188 & 0.183 \\
\hline $\mathrm{N}$ & $52.7 \pm 4.0$ & $53.6 \pm 2.5$ & $53.6 \pm 4.1$ & 0.301 & 0.931 & 0.174 \\
\hline I & $50.3 \pm 5.0$ & $52.3 \pm 2.5$ & $52.1 \pm 0.1$ & 0.094 & 0.264 & 0.330 \\
\hline FAZ $\left(\mathrm{mm}^{2}\right)$ & $0.30 \pm 0.11$ & $0.31 \pm 0.10$ & $0.29 \pm 0.11$ & 0.530 & 0.285 & 0.681 \\
\hline
\end{tabular}

SD: standard deviation; SCP: superficial capillary plexus; T: temporal; S: superior; N: nasal; I: inferior; DCP: deep capillary plexus; FAZ: foveal avascular zone.

were no significant differences in DCP vessel density and the FAZ area among the three groups $(P>0.141)$.

The LogMAR BCVA for the 22 amblyopic eyes at enrolment was $0.42 \pm 0.31$ (range: $0.2-1.3$ ). The follow-up time was $7.5 \pm 4.4$ months (range: $3-12$ months), at which time BCVA had improved to LogMAR $0.27 \pm 0.29$ ( $P=0.001$, paired $t$-test). The SE before treatment in the amblyopic eyes and fellow eyes was $4.44 \pm 1.89 \mathrm{D}$ and $0.70 \pm 0.87 \mathrm{D}$, respectively. After treatment, it was $3.79 \pm 1.93$ $\mathrm{D}$ and $0.41 \pm 0.76 \mathrm{D}$, respectively. Both were significantly less hyperopic than before amblyopia treatment $(P=0.001$ and 0.019 , respectively). As expected, based on the decrease of hyperopic SE and increase of age, the ALs of the amblyopic and fellow eyes after treatment $(22.16 \pm 1.14 \mathrm{~mm}$ and $23.26 \pm 0.98 \mathrm{~mm}$, respectively) were longer than before treatment $(21.75 \pm 0.96 \mathrm{~mm}$ and $23.05 \pm 0.90 \mathrm{~mm}$, respectively) ( $P=0.038$ and $<0.001$, respectively).

For all amblyopic subjects, the densities of the foveal and parafoveal SCPs after amblyopia treatment were $23.0 \pm 5.6 \%$ and $50.0 \pm 3.6 \%$, respectively. The SCP foveal and parafoveal densities in the fellow eyes after amblyopia treatment were $24.6 \pm 5.8 \%$ and $51.8 \pm 2.2 \%$, respectively. For the DCP, the foveal and parafoveal densities in amblyopic eyes after treatment were $32.5 \pm 7.6 \%$ and $52.4 \pm 2.9 \%$, respectively, and the densities in the fellow eyes after treatment were $32.5 \pm 6.5 \%$ and $52.9 \pm 2.7 \%$, respectively. The interaction between eyes and amblyopia treatment was not significant for either the foveal or the parafoveal vessel SCP densities ( $P=0.614$ and 0.138 , respectively, factorial repeated-measures ANOVA). Similarly, the interaction between eyes and amblyopia treatment was not significant for the DCP densities in either the fovea or parafovea $(P=0.570$ and 0.996, respectively). As described above, the parafoveal SCP vessel density in amblyopic eyes, $49.0 \pm 4.1 \%$, was less than in fellow eyes, $51.8 \pm 2.2 \% \quad(P=0.012$, paired $t$-test $)$ before treatment. However, after treatment, the difference between them was no longer significant $(P=0.187)$.
Among the amblyopic subjects, 36\% (8) had no improvement in BCVA during treatment, while 64\% (14) improved from LogMAR $0.42 \pm 0.27$ before treatment to $0.18 \pm 0.18$ afterwards (Table 3 ). The median age of subjects with BCVA improvement was 7 (IQR 6-8.25) years old, which was younger than that of subjects without BCVA improvement (median 9 years old, IQR 8-9.75 years, $P=0.034)$. There were no significant differences in the initial BCVA or SEs between the two groups $(P=0.725$ and 0.958 , respectively). The duration of treatment, $8.28 \pm 4.50$ months, for subjects with improved BCVA was not significantly different from the treatment duration of those who had no improvement in BCVA, 6.06 \pm 4.02 months $(P=0.249)$. However, the combined factors of age and treatment duration may partially account for some patients having improved BCVA while the others did not.

The foveal and parafoveal SCP vessel density changes in the fellow eyes of subjects with or without BCVA improvement in the amblyopic eyes were virtually the same at each quadrant position where the macular SCP vessel densities tended to decrease (Table 3 ). The parafoveal vessel densities in the amblyopic eyes with no BCVA improvement changed in a similar manner. At each position in the parafovea, the vessel density tended to decrease, though again, the changes were not statistically significant. However, the fovea showed virtually no change. On the other hand, the amblyopic eyes with improved BCVA tended to have an increase in SCP vessel density in each parafoveal position, while the SCP density tended to decrease in the corresponding fellow eyes (Table 3).

Multiple linear regression analysis showed that older age and greater foveal SCP vessel density were negatively correlated with $\mathrm{BCVA}$ improvement $(\beta=-0.057, P=0.017$; $\beta=-0.033, P=0.031$, respectively, Table 4$)$. This suggests that lower macular SCP vessel density combined with other factors is predictive of a greater therapeutic effect of patching therapy. 
TABle 3: Parameters and macular vessel densities in subjects with VA improvement and without VA improvement.

\begin{tabular}{|c|c|c|c|c|c|c|c|c|}
\hline & \multicolumn{4}{|c|}{ Subjects with VA improvement } & \multicolumn{4}{|c|}{ Subjects without VA improvement } \\
\hline & \multicolumn{2}{|c|}{ Amblyopic eye } & \multicolumn{2}{|c|}{ Fellow eye } & \multicolumn{2}{|c|}{ Amblyopic eye } & \multicolumn{2}{|c|}{ Fellow eye } \\
\hline & $\begin{array}{c}\text { Before } \\
\text { treatment }\end{array}$ & $\begin{array}{c}\text { After } \\
\text { treatment }\end{array}$ & $\begin{array}{c}\text { Before } \\
\text { treatment }\end{array}$ & $\begin{array}{c}\text { After } \\
\text { treatment }\end{array}$ & $\begin{array}{c}\text { Before } \\
\text { treatment }\end{array}$ & $\begin{array}{c}\text { After } \\
\text { treatment }\end{array}$ & $\begin{array}{c}\text { Before } \\
\text { treatment }\end{array}$ & $\begin{array}{c}\text { After } \\
\text { treatment }\end{array}$ \\
\hline SE (D) & $4.34 \pm 2.1$ & $3.59 \pm 2.0$ & $0.74 \pm 0.74$ & $0.53 \pm 0.69$ & $4.63 \pm 1.63$ & $4.16 \pm 1.89$ & $0.64 \pm 1.10$ & $0.21 \pm 0.85$ \\
\hline $\mathrm{AL}(\mathrm{mm})$ & $21.78 \pm 0.96$ & $22.19 \pm 1.26$ & $22.91 \pm 0.91$ & $23.09 \pm 1.01$ & $21.71 \pm 1.04$ & $22.19 \pm 1.25$ & $23.29 \pm 0.89$ & $23.36 \pm 0.91$ \\
\hline $\begin{array}{l}\text { VA } \\
\text { (LogMAR) }\end{array}$ & $0.42 \pm 0.27$ & $0.18 \pm 0.18$ & $-0.00 \pm 0.02$ & $-0.01 \pm 0.02$ & $0.43 \pm 0.39$ & $0.43 \pm 0.39$ & $-0.01 \pm 0.03$ & $-0.01 \pm 0.03$ \\
\hline $\begin{array}{l}\text { Retinal } \\
\text { region }\end{array}$ & \multicolumn{8}{|c|}{ Macular vessel density, mean \pm SD (\%) } \\
\hline \multicolumn{9}{|l|}{ SCP } \\
\hline Fovea & $23.3 \pm 5.2$ & $22.4 \pm 5.7$ & $24.1 \pm 6.4$ & $21.6 \pm 5.4$ & $23.9 \pm 4.9$ & $24.1 \pm 5.6$ & $25.5 \pm 4.9$ & $23.9 \pm 4.9$ \\
\hline Parafovea & $48.3 \pm 4.5$ & $49.6 \pm 2.8$ & $51.4 \pm 2.4$ & $50.9 \pm 1.9$ & $51.5 \pm 1.5$ & $50.6 \pm 4.9$ & $52.4 \pm 1.7$ & $51.5 \pm 1.5$ \\
\hline $\mathrm{T}$ & $47.0 \pm 5.3$ & $48.5 \pm 3.1$ & $50.3 \pm 2.8$ & $49.8 \pm 2.1$ & $50.6 \pm 1.9$ & $48.0 \pm 7.5$ & $51.8 \pm 1.8$ & $50.6 \pm 1.9$ \\
\hline $\mathrm{S}$ & $50.2 \pm 3.8$ & $51.3 \pm 2.6$ & $52.3 \pm 3.2$ & $51.7 \pm 2.5$ & $52.3 \pm 2.2$ & $51.5 \pm 4.0$ & $53.2 \pm 2.2$ & $52.3 \pm 2.2$ \\
\hline $\mathrm{N}$ & $47.2 \pm 4.7$ & $48.8 \pm 4.0$ & $50.7 \pm 2.3$ & $50.2 \pm 1.8$ & $51.0 \pm 1.6$ & $50.8 \pm 4.4$ & $51.9 \pm 1.6$ & $51.0 \pm 1.6$ \\
\hline I & $49.2 \pm 4.8$ & $49.7 \pm 3.2$ & $52.2 \pm 2.7$ & $51.8 \pm 2.5$ & $52.1 \pm 2.3$ & $52.1 \pm 4.8$ & $52.7 \pm 2.8$ & $52.1 \pm 2.3$ \\
\hline \multicolumn{9}{|l|}{ DCP } \\
\hline Fovea & $32.0 \pm 6.8$ & $32.0 \pm 8.7$ & $32.1 \pm 7.5$ & $32.1 \pm 7.3$ & $32.4 \pm 5.1$ & $33.2 \pm 5.5$ & $33.0 \pm 4.7$ & $32.6 \pm 4.5$ \\
\hline Parafovea & $52.6 \pm 4.9$ & $52.3 \pm 3.3$ & $53.3 \pm 2.6$ & $54.4 \pm 2.3$ & $50.0 \pm 2.3$ & $52.4 \pm 2.1$ & $52.2 \pm 2.9$ & $52.3 \pm 2.1$ \\
\hline $\mathrm{T}$ & $52.9 \pm 4.4$ & $52.5 \pm 3.5$ & $52.6 \pm 4.0$ & $53.4 \pm 4.5$ & $50.3 \pm 2.1$ & $51.7 \pm 4.3$ & $52.5 \pm 2.4$ & $53.0 \pm 2.3$ \\
\hline S & $52.0 \pm 6.2$ & $52.4 \pm 4.0$ & $53.6 \pm 3.2$ & $54.4 \pm 2.6$ & $50.6 \pm 2.5$ & $52.9 \pm 2.4$ & $52.3 \pm 3.7$ & $52.4 \pm 2.9$ \\
\hline $\mathrm{N}$ & $53.9 \pm 4.2$ & $53.1 \pm 2.7$ & $54.2 \pm 2.3$ & $54.7 \pm 2.5$ & $50.7 \pm 2.6$ & $53.1 \pm 2.1$ & $52.6 \pm 2.7$ & $52.8 \pm 1.5$ \\
\hline I & $51.5 \pm 5.7$ & $51.0 \pm 4.1$ & $52.9 \pm 1.9$ & $53.6 \pm 2.5$ & $48.4 \pm 3.1$ & $52.3 \pm 4.1$ & $51.3 \pm 3.2$ & $51.2 \pm 2.9$ \\
\hline FAZ $\left(\mathrm{mm}^{2}\right)$ & $0.30 \pm 0.12$ & $0.30 \pm 0.12$ & $0.31 \pm 0.12$ & $0.32 \pm 0.13$ & $0.30 \pm 0.08$ & $0.29 \pm 0.07$ & $0.30 \pm 0.07$ & $0.31 \pm 0.07$ \\
\hline
\end{tabular}

SE: spherical equivalent; D: diopter; LogMAR: logarithm (minimum angle of resolution); AL: axial length; SCP: superficial capillary plexus; T: temporal; S: superior; N: nasal; I: inferior; DCP: deep capillary plexus; FAZ: foveal avascular zone.

TABLE 4: Multiple linear regression analysis for associations of the BCVA improvement with age, refraction, BCVA at initial visit, duration of treatment, and macular vessel density.

\begin{tabular}{lcc}
\hline Characteristics & \multicolumn{2}{c}{ BCVA improvement } \\
& $\beta(95 \%$ CI $))$ & $P$ \\
\hline Age $(y)$ & $-0.057(-0.102,0.012)$ & 0.017 \\
Initial SE (D) & $0.003(-0.040,0.047)$ & 0.875 \\
Initial BCVA (LogMAR) & $0.107(-0.163,0.376)$ & 0.409 \\
Treatment duration & $0.006(-0.011,0.022)$ & 0.457 \\
Vessel density & & \\
$\quad$ Foveal SCP & $-0.033(-0.062,-0.003)$ & 0.031 \\
$\quad$ Parafoveal SCP & $0.012(-0.009,0.034)$ & 0.244 \\
$\quad$ Foveal DCP & $0.022(-0.002,0.045)$ & 0.069 \\
$\quad$ Parafoveal DCP & $0.007(-0.012,0.026)$ & 0.442 \\
\hline
\end{tabular}

SE: spherical equivalent; D: diopter; LogMAR: logarithm (minimum angle of resolution); BCVA: best-corrected visual acuity; SCP: superficial capillary plexus; DCP: deep capillary plexus.

\section{Discussion}

Our OCTA data showed that amblyopic eyes have significantly lower vessel density in the SCP of the macula, especially in the parafoveal region. The vessel density measurements were consistent with our previous report [9]. For amblyopic eyes in which the BCVA improved with treatment, the parafoveal macular SCP vessel density increased relative to the fellow eye, and it increased even more relative to the amblyopic eyes in which BCVA did not improve due to treatment. Age and pretreatment macula vessel density were independently negatively correlated with BCVA improvement.

Several studies have reported reduced macular blood flow in amblyopic patients. For example, Lonngi et al. [10] showed that the macular SCP and DCP vessel densities in unilateral amblyopes were less than in the fellow eyes. Yilmaz et al. [12] reported the same results in patients with strabismus amblyopia. Sobral et al. [11] found that lower vessel density in unilateral amblyopic eyes occurred only in the SCP. Recently, our group evaluated macular vessel density in patients with anisometropic, strabismic, and binocular amblyopia [9]. We found that macular vessel density is lower in anisometropic amblyopia, and to a lesser extent, in the other amblyopias. As a result, the present longitudinal study focused just on anisometropic amblyopia. Both Chen et al. [9] and Cheung et al. [17] reported a positive correlation between macular perfusion and the thickness of the macular retina.

Several researchers have measured retinal thickness during amblyopia treatment, and the results among them are not consistent. Pang et al. [18] measured macular thickness in 17 myopic anisometropic amblyopic children during a 32week follow-up period. They reported that the foveal thickness in the amblyopic eyes was significantly reduced; however, there were no significant changes in the parafoveal thickness after amblyopia treatment even though BCVA improved by 3 lines. On the other hand, Nishi et al. [7] did not find significant changes in foveal thickness. However, they did report an increase in the photoreceptor outer 
segment layer length in 21 hyperopic anisometropic amblyopic children during treatment for 1 year, during which BCVA improved by 3 lines. In addition, Chen et al. [19] reported no difference in macular thickness between untreated and treated amblyopes. Tugcu et al. [20] studied macular thickness in the amblyopic eyes of 14 children with persistent amblyopia and 18 children with resolved amblyopia and reported no difference in foveal thickness between the two groups. They declared that OCT examinations could not explain why some cases were resistant to amblyopia treatment.

Age influenced the process of BCVA improvement, which is consistent with previous studies. For example, Chen et al. [21] retrospectively found that the BCVA of 217 binocular amblyopic children aged 6 years or younger improved significantly more than in children aged 7 to 10 years. They also reported that BCVA improvement was negatively correlated with age, which was consistent with our results. Interestingly, macular vessel density was negatively correlated with BCVA improvement, which means children with lower vessel density will have larger BCVA improvements. This is consistent with our findings that macular vessel density in amblyopic eyes was smaller than in fellow eyes, and the difference between the two eyes was reduced during treatment. Evidently, patients with lower vessel density in the amblyopic eye experienced greater treatment-dependent increases in vessel density, thereby reducing the difference in vessel density between the two eyes. Similarly, Sina Salehi and Sean [22] evaluated the relationship between characteristics of visual evoked potentials (VEP) and the outcome of occlusion therapy. They reported that the P100 latency time correlates negatively with eventual improvement in BCVA. Thus, they proposed that the VEP P100 latency response in an amblyopic eye can predict how well a child will respond to occlusion therapy. Our results are consistent with this hypothesis; however, further research in which both VEP and OCTA are performed on the same patients is necessary to test this idea.

The standard optotype BCVA of anisometropic amblyopes is reduced primarily due to resolution acuity and contrast sensitivity losses within the primary visual system between the retina and the striate cortex [23, 24]. Numerous studies have shown that when a group of neurons atrophy, the incoming synapses will atrophy or become less responsive [25]. In the case of amblyopia, retinal ganglion cells would be expected to become less active through transneuronal degeneration from LGN atrophy [26]. Thus, the involvement of ganglion cells in amblyopic visual loss should not be surprising. We found only a small change in macular vessel density in relation to visual improvement. However, we are not certain if a much stronger recovery of neurons in the striate cortex or LGN could induce a transneuronal degeneration change in the retinal ganglion cells. Animal studies are needed to detect this change throughout the visual system in association with vision recovery from amblyopia.

There are several limitations in our study. First, to obtain good quality OCT scan images, the subjects had to be beyond the traditional end of the sensitive period for amblyopic treatment. Consequently, there were relatively few subjects in the acceptable age range. Second, because of poor parental compliance, the follow-up duration varied in length, and the average duration time was relatively short.

\section{Conclusion}

The vascular density in the macular SCP in the parafoveal area of hyperopic anisometropic amblyopic eyes was reduced relative to fellow nonamblyopic eyes and to normal control eyes. After 7.5 months of amblyopia therapy, in amblyopic eyes with improved BCVA, the parafoveal macular SCP vessel density increased relative to the amblyopic eyes in which BCVA did not improve. In addition, the baseline parafoveal SCP vascular density and the initial age of the patients were correlated with the improvement in BCVA. Thus, the macular vascular density appears to be a part of the hyperopic anisometropic amblyopia syndrome. The understanding of the relationship between macular vascular density and hyperopic anisometropic amblyopia is an important change from current models of amblyopia and deserves to be tested in studies with larger sample sizes. Furthermore, other factors such as patching compliance and stereopsis can also be important confounding variables which may have an impact on efficiency of amblyopic treatment. More possible factors should be designed in future studies.

\section{Data Availability}

The original data, figures, and tables that were used to support the findings of this study are available from the corresponding author upon request.

\section{Conflicts of Interest}

The authors declare that they have no conflicts of interest.

\section{Acknowledgments}

The authors thank Shenghai Huang, Yuanyuan Zhou, Meiping Xu, Jinjing Zhou, Jinling Xu, MD, Suzhong Xu, and Hui Chen at Wenzhou Medical University Affiliated Hospital, for their collaboration with the authors on this study. This work was supported by the Zhejiang Provincial Natural Science Foundation of China under Grant no. LQ18H120005.

\section{References}

[1] K. Simons, "Amblyopia characterization, treatment, and prophylaxis," Survey of Ophthalmology, vol. 50, no. 2, pp. 123-166, 2005.

[2] D. H. Hubel, T. N. Wiesel, and S. LeVay, "Plasticity of ocular dominance columns in monkey striate cortex," Philosophical Transactions of the Royal Society of London. Series B, Biological Sciences, vol. 278, no. 961, pp. 377-409, 1977.

[3] G. K. von Noorden and P. R. Middleditch, "Histology of the monkey lateral geniculate nucleus after unilateral lid closure and experimental strabismus: further observations," 
Investigative Ophthalmology \& Visual Science, vol. 14, no. 9, pp. 674-683, 1975.

[4] C. E. Al-Haddad, G. M. E. L. Mollayess, C. G. Cherfan, D. F. Jaafar, and Z. F. Bashshur, "Retinal nerve fibre layer and macular thickness in amblyopia as measured by spectraldomain optical coherence tomography," British Journal of Ophthalmology, vol. 95, no. 12, pp. 1696-1699, 2011.

[5] S. C. Huynh, C. Samarawickrama, X. Y. Wang et al., "Macular and nerve fiber layer thickness in amblyopia," Ophthalmology, vol. 116, no. 9, pp. 1604-1609, 2009.

[6] Y. Pang, G. W. Goodfellow, C. Allison, S. Block, and K. A. Frantz, "A prospective study of macular thickness in amblyopic children with unilateral high myopia," Investigative Opthalmology \& Visual Science, vol. 52, no. 5, pp. 2444-2449, 2011.

[7] T. Nishi, T. Ueda, T. Hasegawa, K. Miyata, and N. Ogata, "Retinal thickness in children with anisohypermetropic amblyopia," British Journal of Ophthalmology, vol. 99, no. 8, pp. 1060-1064, 2015.

[8] B. Demirayak, A. Vural, I. U. Onur, F. S. Kaya, and F. U. Yigit, "Analysis of macular vessel density and foveal avascular zone using spectral-domain optical coherence tomography angiography in children with amblyopia," Journal of Pediatric Ophthalmology \& Strabismus, vol. 56, no. 1, pp. 55-59, 2019.

[9] W. Chen, J. Lou, F. Thorn et al., "Retinal microvasculature in amblyopic children and the quantitative relationship between retinal perfusion and thickness," Investigative Opthalmology \& Visual Science, vol. 60, no. 4, pp. 1185-1191, 2019.

[10] M. Lonngi, F. G. Velez, I. Tsui et al., "Spectral-domain optical coherence tomographic angiography in children with amblyopia," JAMA Ophthalmology, vol. 135, no. 10 , pp. 1086-1091, 2017.

[11] I. Sobral, T. M. Rodrigues, M. Soares et al., "OCT angiography findings in children with amblyopia," Journal of American Association for Pediatric Ophthalmology and Strabismus, vol. 22, no. 4, pp. 286-289, 2018.

[12] I. Yilmaz, O. B. Ocak, B. S. Yilmaz, A. Inal, B. Gokyigit, and M. Taskapili, "Comparison of quantitative measurement of foveal avascular zone and macular vessel density in eyes of children with amblyopia and healthy controls: an optical coherence tomography angiography study," Journal of American Association for Pediatric Ophthalmology and Strabismus, vol. 21, no. 3, pp. 224-228, 2017.

[13] E. Cinar, B. Yuce, F. Aslan, and G. Erbakan, "Comparison of retinal vascular structure in eyes with and without amblyopia by optical coherence tomography angiography," Journal of Pediatric Ophthalmology \& Strabismus, vol. 57, no. 1, pp. 48-53, 2020.

[14] D. K. Wallace, C. L. Morse, M. Melia et al., "Pediatric eye evaluations preferred Practice pattern," Ophthalmology, vol. 125, no. 1, pp. P184-P227, 2018.

[15] S. S. Gao, Y. Jia, L. Liu et al., "Compensation for reflectance variation in vessel density quantification by optical coherence tomography angiography," Investigative Opthalmology \& Visual Science, vol. 57, no. 10, pp. 4485-4492, 2016.

[16] J. Yu, R. Gu, Y. Zong et al., "Relationship between retinal perfusion and retinal thickness in healthy subjects: an optical coherence tomography angiography study," Investigative Opthalmology \& Visual Science, vol. 57, no. 9, Article ID OCT204, 2016.

[17] C. Y. Cheung, J. Li, N. Yuan et al., "Quantitative retinal microvasculature in children using swept-source optical coherence tomography: the Hong Kong Children eye study,"
British Journal of Ophthalmology, vol. 103, no. 5, pp. 672-679, 2019.

[18] Y. Pang, K. A. Frantz, S. Block, G. W. Goodfellow, and C. Allison, "Effect of amblyopia treatment on macular thickness in eyes with myopic anisometropic amblyopia," Investigative Opthalmology \& Visual Science, vol. 56, no. 4, pp. 2677-2683, 2015.

[19] W. Chen, J. Chen, J. Huang, J. Xu, F. Zhang, and F. Lu, "Comparison of macular and retinal nerve fiber layer thickness in untreated and treated binocular amblyopia," Current Eye Research, vol. 38, no. 12, pp. 1248-1254, 2013.

[20] B. Tugcu, B. Araz-Ersan, E. T. Erdogan et al., "Structural and functional comparison of the persistent and resolved amblyopia," Documenta Ophthalmologica, vol. 128, no. 2, pp. 101-109, 2014.

[21] W. Chen, J. Chen, F. Zhang, X. Zhu, and F. Lu, "Visual outcome in isoametropic amblyopic children with high hyperopia and the effect of therapy on retinal thickness," American Journal of Ophthalmology, vol. 155, no. 3, pp. 536-543 e1, 2013.

[22] O. Sina Salehi and P. D. Sean, "Visual evoked potential latency predicts improvement in amblyopia following occlusion therapy," Journal of American Association for Pediatric Ophthalmology and Strabismus, vol. 17, no. 1, p. e27, 2013.

[23] D. M. Levi and S. Klein, "Hyperacuity and amblyopia," $\mathrm{Na}$ ture, vol. 298, no. 5871, pp. 268-270, 1982.

[24] D. M. Levi, S. P. McKee, and J. A. Movshon, "Visual deficits in anisometropia," Vision Research, vol. 51, no. 1, pp. 48-57, 2011.

[25] H. E. Pearson and T. P. Thompson, "Atrophy and degeneration of ganglion cells in central retina following loss of postsynaptic target neurons in the dorsal lateral geniculate nucleus of the adult cat," Experimental Neurology, vol. 116, no. 2, pp. 163-171, 1992.

[26] B. M. Hooks and C. Chen, "Vision triggers an experiencedependent sensitive period at the retinogeniculate synapse," Journal of Neuroscience, vol. 28, no. 18, pp. 4807-4817, 2008. 